\title{
Recent Results from HERA
}

\author{
Andrea Parenti \\ Università degli Studi di Padova and INFN Sezione di Padova, \\ via Marzolo 8, 35131 Padova, Italy. \\ parenti@pd.infn.it
}

\section{Introduction}

The HERA collider located in Hamburg, Germany, is a unique facility which collides protons of $920 \mathrm{GeV} 1$ and electrons (or positrons) of $27.5 \mathrm{GeV}$.

The $e^{ \pm} p$ interactions proceed via a $\gamma / Z^{0}$ exchange in the neutral current (NC) reaction, or via a $W^{ \pm}$exchange in the charged current (CC) interaction.

HERA is taking data since 1992; after a shutdown in 2000, data taking has restarted in 2002 with a five-fold increase in the luminosity. The first running period is referred to as HERA-I, whereas the second one is referred to as HERA-II.

In this report I will review some recent results obtained by the $\mathrm{H} 1$ and ZEUS experiments at the HERA collider. Many different processes can be studied at HERA; this selection reflects my personal taste and many other relevant results have been omitted.

\section{Results}

\section{Structure Functions and Parton Distribution Functions}

The cross section for the $e p \rightarrow e X$ process with unpolarised lepton beams depends on the proton structure functions $F_{2}, F_{L}$ and $F_{3}$; the prominent contribution comes from $F_{2}$. HERA is the ideal machine to study proton structure functions, since the accessible kinematical region is much larger than in hadronic colliders and fixed target experiments (see Fig. 1).

$F_{2}$ was measured by using HERA-I data [1, 2] in the region $6.32 \times 10^{-5}<$ $x<0.65$ and $1<Q^{2}<30000 \mathrm{GeV}^{2}$ with an uncertainty as little as 2 $3 \%$ (see Fig. 1). The longitudinal structure function $F_{L}$ has been measured as well, though with worse precision [3]. $F_{3}$, the parity violating part of the

\footnotetext{
${ }^{1}$ Until 1998, the energy of the proton beam was $820 \mathrm{GeV}$.
} 

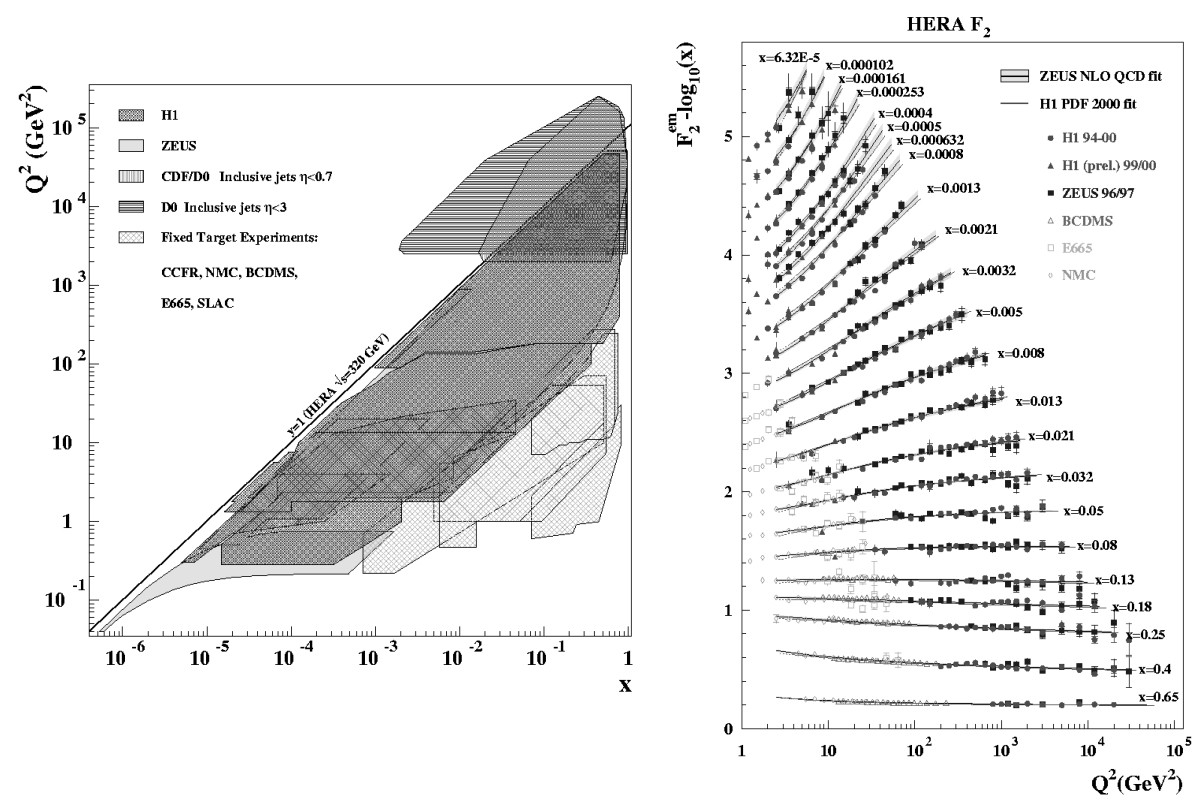

Fig. 1. The $x-Q^{2}$ kinematical plane and the accessible regions at hadronic colliders, in fixed target experiments and at HERA (left). The structure function $F_{2}$ versus $Q^{2}$ at fixed values of $x$, compared with a fit based on DGLAP equations (right).

interaction, is measurable as a difference in $e^{-} p$ and $e^{+} p \mathrm{NC}$ differential cross sections. A measurement from ZEUS [4] - using $e^{-} p$ collisions from HERA-II and $e^{+} p$ from HERA-I - has been presented at DIS 2006; this measurement has a much lower statistical uncertainty than with HERA-I data only.

The charm and beauty contributions to $F_{2}$ have been extracted from HERA-I data by H1 [5, [6] and ZEUS [7]. H1 used the impact parameter significance in order to evaluate the heavy quark fraction in the sample; in the region $Q^{2}>150 \mathrm{GeV}^{2}$ and $0.1<y<0.7$ the charm and beauty contributions to $F_{2}$ are $18.3 \pm 3.0 \%$ and $2.72 \pm 0.74 \%$, respectively. ZEUS at the time did not have any vertex detector and therefore identified only charm by the decay chain $D^{*} \rightarrow D^{0} \pi_{s}, D^{0} \rightarrow K \pi$. The charm contribution to $F_{2}$ goes up to $\sim 30 \%$ at $Q^{2}=500 \mathrm{GeV}^{2}$ and $x=0.012$. A vertex detector was installed in the ZEUS detector before HERA-II phase, therefore ZEUS will be able to evaluate $F_{2}^{b \bar{b}}$ with the new data.

The proton parton distribution functions (PDFs) were extracted by ZEUS [8] from the $\mathrm{NC}$ and $\mathrm{CC}$ cross sections, jet production in $e^{+} p$ collisions, dijet production in $\gamma p$ collisions, by using only HERA-I data and no other data from different experiments (the so-called ZEUS-JET fit). The results are already competitive to MRST and CTEQ fits. The importance of the ZEUS-JET fit 
is that the uncertainty is dominated by statistics and will be reduced significantly by the use of HERA-II data, whereas in MRST and CTEQ the uncertainty comes mainly from systematics.

\section{First Results with Polarised Lepton Beam}

The leptons at HERA acquire spontaneously a transverse polarisation due to the synchrotron light emission. In 2003 spin rotators were installed before $\mathrm{H} 1$ and ZEUS interaction points, transforming the transverse polarisation in longitudinal. The $\mathrm{CC}$ cross section depends linearly on the degree of polarisation of the beam, $P_{e}: \sigma_{\mathrm{CC}}^{e^{ \pm} p}=\left(1 \pm P_{e}\right) \sigma_{\mathrm{CC} \text {,unpol }}^{e^{ \pm} p}$.

H1 and ZEUS have measured [9, 10] the total cross section of the CC process for $e^{+} p$ collisions and different values of polarisation and found a nice agreement with the expected $\left(1+P_{e}\right)$ dependence.

The NC dependence on $P_{e}$ is more complicated; ZEUS has measured [10] the $d \sigma / d Q^{2}$ in $e^{+} p$ collisions with positive and negative values of the polarisation and found again a nice agreement with the Standard Model prediction.

\section{Isolated Leptons at High $P_{T}$}

Events with missing $P_{T}$ and the production of an isolated, high- $P_{T}$ lepton come mainly from the process $e p \rightarrow e W X, W \rightarrow l \nu(l=e, \mu, \tau)$; investigations of the process with $l=e, \mu$ have been performed by both the H1 and ZEUS experiments [11]. H1 observed an excess of high- $P_{T}$ isolated leptons in HERA-I $e^{+} p$ data with respect to the expectation from $W$ production; this excess was not seen by ZEUS. The analysis of HERA-II data confirms the HERA-I results: $\mathrm{H} 1$ observed 15 events in 1994-2004 $e^{+} p$ data having hadronic $p_{T}>25 \mathrm{GeV}\left(4.6 \pm 0.8\right.$ expected), whereas no excess was seen in $e^{-} p$ collisions and by ZEUS (in both $e^{ \pm} p$ ). These results are not yet understood.

\section{Measurement of $\alpha_{S}$ at HERA}

The strong coupling constant, $\alpha_{S}$, appears in all QCD processes: $\alpha_{S}$ can therefore be extracted from many observables.

A precise estimation of $\alpha_{S}$ at $M_{Z}$ scale has been made [12 by averaging the measurements made at HERA (e.g. jet cross section, event shape, jet multiplicity, etc.); the energy dependence of $\alpha_{S}$ was measured, too. The uncertainty of HERA determination is dominated by the theoretical part, whereas the experimental part is already better than the world average total uncertainty.

\section{Search for Pentaquarks at HERA}

A number of experiments observed a narrow baryon resonance with positive strangeness, mass around $1530 \mathrm{MeV}$, decaying to $n K^{+}$or $p(\bar{p}) K_{S}^{0}$. The signals are consistent with an exotic state having quark content $u u d d \bar{s}$, called $\Theta^{+}$. Other experiments did not observe any signal. 
ZEUS observed a peak [13] in the $p(\bar{p}) K_{S}^{0}$ invariant mass spectrum in HERA-I data at $M=1522 \pm 6 \mathrm{MeV} ; 221 \pm 48$ events above the background were seen. In a similar search [14] H1 did not observe any excess over the background.

Since the $\Theta^{+}$belongs to an hypotetical antidecuplet of pentaquarks with spin- $\frac{1}{2}$, two more exotic states are foreseen: $\Xi_{3 / 2}^{--}$and $\Xi_{3 / 2}^{+}$with quark content

$d d s s \bar{u}$ and $u u s s \bar{d}$, respectively. NA49 at CERN SPS reported the observation of $\Xi_{3 / 2}^{--}$and $\Xi_{3 / 2}^{0}$, whereas searches made by other experiments were negative. ZEUS performed such a search but did not observe any signal [15].

The anti-charmed pentaquark $\Theta_{c}^{0}(u u d d \bar{c})$ has been observed by H1 [16]: a peak of $50.6 \pm 11.2$ events was seen in the $D^{*-} p$ and $D^{*+} \bar{p}$ decay channels. However, ZEUS has not observed any excess over the background 17].

No final conclusion can be drawn on pentaquarks.

\section{Conclusion}

In this report a review of recent results from HERA has been presented.

The most relevant result is probably the measurement of the proton struction functions and the extraction of parton distribution functions. Almost all the results were obtained by using HERA-I data only, so we expect a big improvement in the precision when HERA-II data will be included.

\section{References}

[1] C. Adloff et al. Eur. Phys. J., C30:1-32, 2003.

[2] S. Chekanov et al. Eur. Phys. J., C21:443-471, 2001.

[3] E. M. Lobodzinska. hep-ph/0311180.

[4] V. Chekelian, C. Gwenlan, and R. S. Thorne. hep-ph/0607116.

[5] A. Aktas et al. Eur. Phys. J., C45:23-33, 2006.

[6] A. Aktas et al. Eur. Phys. J., C40:349-359, 2005.

[7] S. Chekanov et al. Phys. Rev., D69:012004, 2004.

[8] S. Chekanov et al. Eur. Phys. J., C42:1-16, 2005.

[9] A. Atkas et al. Phys. Lett., B634:173-179, 2006.

[10] S. Chekanov et al. Phys. Lett., B637:210-222, 2006.

[11] J. Ferrando. PoS, HEP2005:319, 2006.

[12] Claudia Glasman. AIP Conf. Proc., 792:689-692, 2005.

[13] S. Chekanov et al. Phys. Lett., B591:7-22, 2004.

[14] Sergei Chekanov. PoS, HEP2005:086, 2006.

[15] S. Chekanov et al. Phys. Lett., B610:212-224, 2005.

[16] A. Atkas et al. Phys. Lett., B588:17, 2004.

[17] S. Chekanov et al. Eur. Phys. J., C38:29-41, 2004. 